\title{
Performance Analysis and Comparison of Full Chip and Half Chip Rate DC and NC Code Acquisition in MIMO DSCDMA over Uncorrelated Rayleigh Wireless Channel
}

\author{
N. Sathish Kumar and K. R. Shankar Kumar \\ Sri Ramakrishna Engineering College, Coimbatore 641022, Tamil Nadu, India \\ Correspondence should be addressed to N. Sathish Kumar, nsk20022002@gmail.com \\ Received 15 January 2011; Accepted 12 March 2011 \\ Academic Editor: G. Tsoulos
}

Copyright $\odot 2011$ N. S. Kumar and K. R. Shankar Kumar. This is an open access article distributed under the Creative Commons Attribution License, which permits unrestricted use, distribution, and reproduction in any medium, provided the original work is properly cited.

This paper presents the performance analysis and comparison of full chip and half chip rate of noncoherent (NC) and differentially coherent (DC) code acquisition scheme in (multiple input-multiple output) MIMO assisted by direct sequence spread spectrum (DS-CDMA) wireless system when communicated over uncorrelated Rayleigh channel. Four schemes are investigated, namely, SISO with full chip rate, SISO with Half chip rate, MIMO with full chip rate, and MIMO with half chip rate by varying the code acquisition technique. The simulation is done in RF signal processing Lab using matlab tool box, and the performance metrics are considered, namely, Bit Error Rate (BER) and mean acquisition time (MAT). The simulation results indicate that DC performance is superior than NC in both full chip and half chip rate and also shown that half chip performance is better than full chip in both DC and NC code acquisition methods.

\section{Introduction}

MIMO technique is employed with spread spectrum to increase capacity and improve the overall performance in the MIMO scheme. In these systems, multiple antennas are used at both ends of the wireless link. This method allows cellular systems to provide users with high data rate services through reliable communication link. It has the ability to combat the fading phenomena in wireless links. Recent research in this method has shown an increase in system diversity and bandwidth efficiency [1]. This technique offers higher capacity to wireless systems, and the capacity increases linearly with the number of antennas. A basic MIMO channel model is depicted in Figure 1 with $M$ transmitter and $N$ receiver antennas. In each use of the MIMO channel, a vector $a=\left(a_{1}, a_{2} \ldots a_{M}\right)^{T}$ of complex numbers is sent and a vector $r=\left(r_{1}, r_{2} \ldots r_{N}\right)^{T}$ of complex numbers is received. We assume an input-output relationship of the form

$$
r=H a+V,
$$

where $H$ is an $N \times M$ matrix representing the scattering effects of the channel called as channel matrix, and $V=$ $\left(v_{1}, v_{2}, v_{N}\right)^{T}$ is the noise vector. Throughout this paper, $H$ is assumed as a random matrix with independent complex Gaussian elements $\left\{h_{i j}\right\}$ with mean 0 , and unit variance is denoted as $h_{i j} \sim C N(0,1)$. Also $V$ is assumed as a complex Gaussian random vector with elements $v_{i} \sim C N\left(0, N_{0}\right)$, and $H$ and $V$ are independent of each other and of the data vector $a$.

\section{DS-CDMA Modulation}

A spread spectrum multiple access technique is one which spreads the bandwidth of the data uniformly for the same transmitted power. Spreading code is a pseudo-random (PN) code which has a narrow pulse codes. In CDMA scheme [2], a locally generated code runs at a much higher rate than the data to be transmitted. In this method, the modulated waveform is spread second time in such a way to produce an expanded wideband signal whose bandwidth is greater 


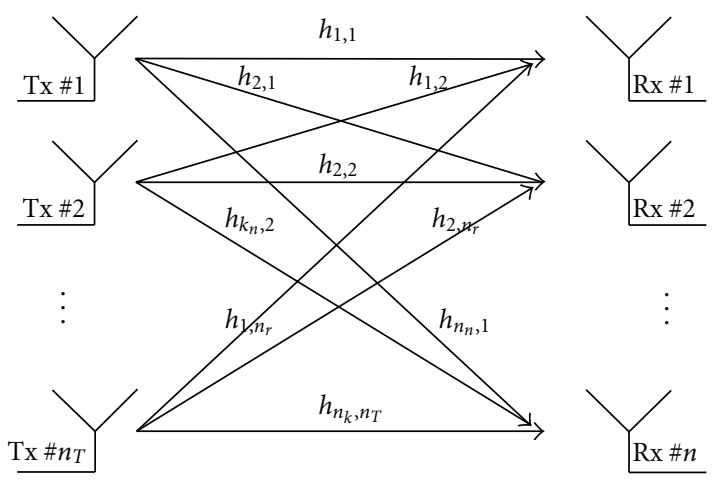

Figure 1: MIMO Model.
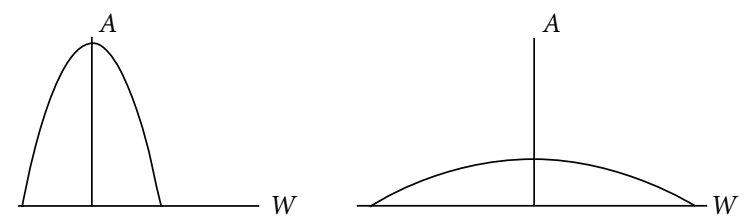

FIgURE 2: Amplitude spectrum before and after spreading.

than the available bandwidth. This signal does not interfere with other signals. such an expansion is achieved using second modulation. Many potential advantages are achieved over conventional systems such as improved interference rejection, high resolution ranging, secured communication and increased capacity, and a better spectral efficiency. The Figure 2 shows the amplitude spectrum before and after spreading.

In DS-SS [3], the spread signal is obtained by multiplying the information signal directly with a wideband PN signal. The information rate is $R=1 / T_{b}$ bits per sec, where $T_{b}$ is one bit interval. The PN signal rate is $1 / T_{c}$ "chips" per sec where $T_{c}$ is one chip duration [4]. One bit interval occupies $(N)$ chips, that is,

$$
N=\frac{T_{b}}{T_{c}}
$$

There are many issues in the conventional DS-CDMA [2] systems which are as given below

(i) If all users transmit almost with identical power then beyond certain point increase of power of every user will not reduce the bit error rate [5].

(ii) If the user transmits with widely different power, then the conventional receiver allows the signal from the powerful user by suppressing the weaker user signal.

(iii) The detection of desired signal is limited by inherent suppression capacity of the system.

\section{System Model}

A finite length tapped with delay line channel model generates the $L$ Rayleigh-faded multipath signals, each is

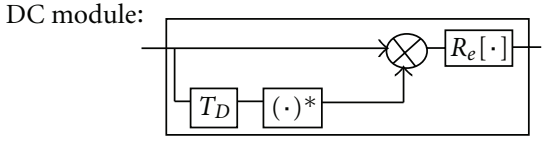

NC module:

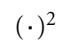

FIgURE 3: DC and NC modules used in the receiver design.

arriving with a time delay $\tau_{l}$ having a tap spacing of one chip duration and half chip duration considered. It is also assumed that the Rayleigh fading is sufficiently slow for the faded envelope to remain constant over $\tau_{D}$ chip intervals. The Neyman-Pearson criterion is adopted $[6,7]$, which leads to a Constant False-Alarm Rate (CFAR). The received signal of the MIMO over the multipath Rayleigh-fading channel can be expressed mathematically as in (4).

The DC and the NC receiver module used in code acquisition scheme using transmit/receive antennas is as shown in Figure 3, and the timing hypothesis test is carried out for binary spreading. The NC module generates its decision variable by accumulating $P \cdot R$ number of independently faded signals observed over a given time interval. In the DC scheme, instead of squaring the summed energy as suggested by the procedures outlined, the channel's output samples accumulated over a full spreading code period is multiplied by the conjugate of the $N$-chip-delayed samples, where $N$ is represented by

$$
\begin{aligned}
& N=\frac{\tau D}{T c}, \\
& r(t)=\sum_{l=1}^{L} \sum_{m=1}^{P} \sum_{n=1}^{R}\left[\alpha_{(l, m, n)} \sqrt{\frac{E c}{P T_{c}}} C\left(t+d T_{c}+\eta\right)\right. \\
& \cdot \omega_{m}\left(t+d T_{c}+\eta\right) e^{\left(2 \pi f t+\phi_{(l, m, n)}\right)} \\
& \left.+I_{k(l, m, n)}(t)\right]
\end{aligned}
$$

where $P=$ number of transmit antennas, $R=$ number of receive antenna, $\alpha_{(l, m, n)}=$ complex-valued envelope of the $(l, m, n)^{\text {th }}$ signal path obeying a Rayleigh magnitude distribution and a uniform phase distribution, $E_{c}=$ pilot signal energy per $\mathrm{PN}$ code chip, $C(t)=$ common $\mathrm{PN}$ sequence having a cell-specific code-phase offset is the code phase offset with respect to the phase of the local code, $T_{c}=$ one chip duration, $w_{m}(t)=$ specific Walsh code assigned to the $m$ th transmit antenna, $f=$ carrier frequency, $\phi=$ carrier phase of a specific user's modulator.

where $k=k^{\text {th }} \psi$ chip's sampling instant, $S_{k(l, m, n)}=\mathrm{a}$ deterministic value, which depends on whether a signal is present or absent. Furthermore, the definition of $W_{1, k(l, m, n)}$, $W_{2, k(l, m, n)}, W_{3, k(l, m, n)}$, and $W_{4, k(l, m, n)}$ which are mutually independent Gaussian random variables having zero means and unit variances.

Equation (4) can be given in a simplified form as

$$
Z_{k(l)}{ }^{\mathrm{DC}}=X_{k(l)}-Y_{k(l)}
$$




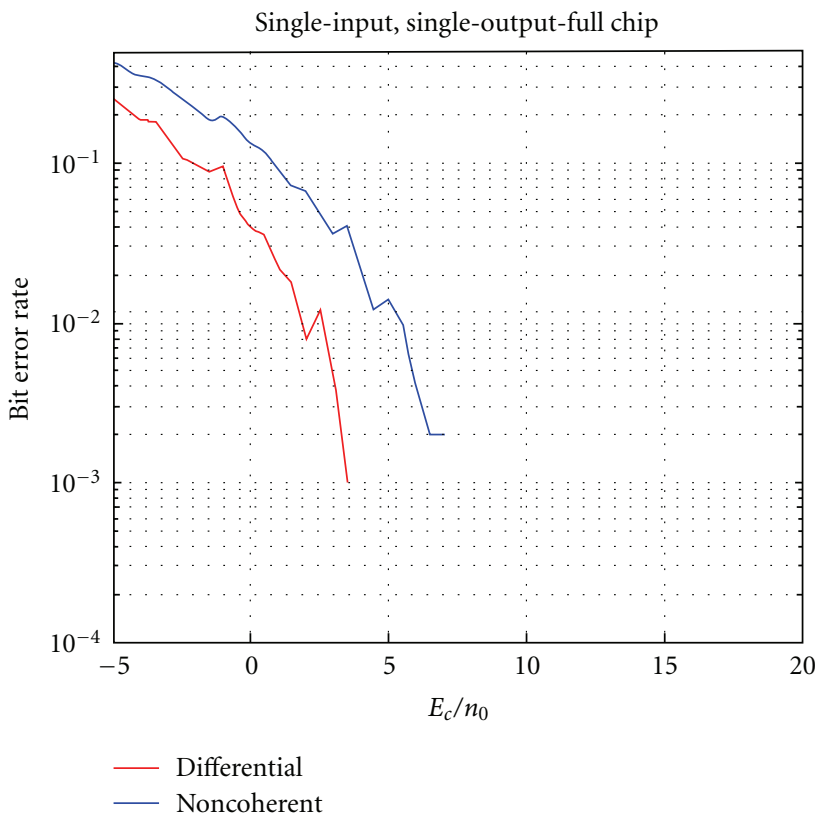

(a)

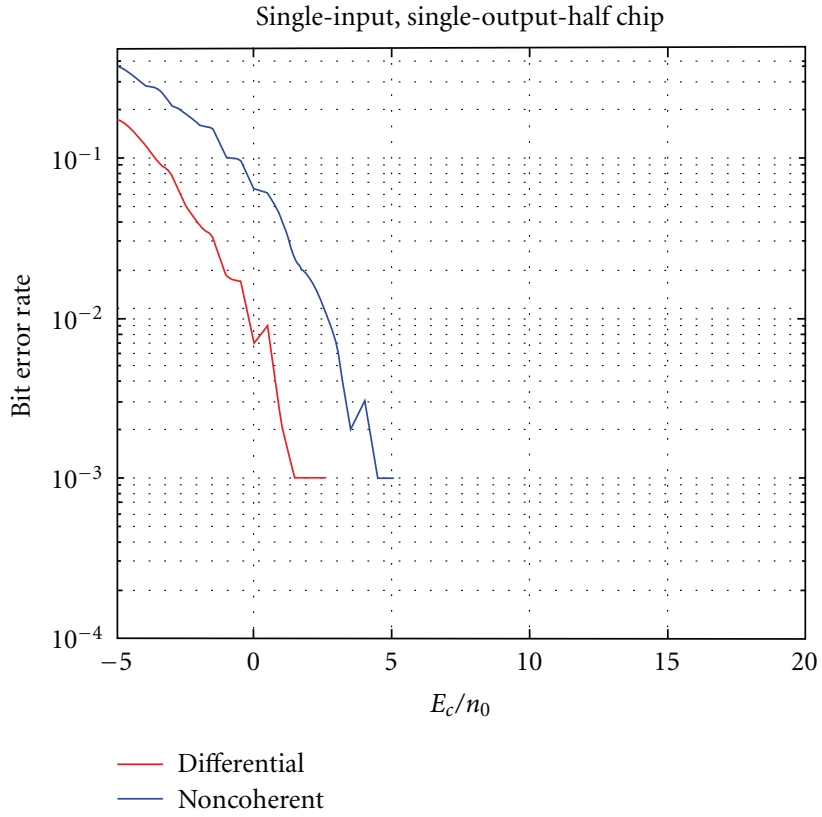

(b)

FIgURE 4: (a, b) BER characteristics of SISO full chip and half chip rate, respectively.

where,

$$
\begin{gathered}
X_{k(l)}=\sum_{m=1}^{P} \sum_{n=1}^{R}\left[\left(\sqrt{\frac{4 E_{c}}{N I_{0} P}} \cdot S_{k(l, m, n)}+W_{1, k(l, m, n)}\right)^{2}+W_{3, k(l, m, n)}^{2}\right], \\
Y_{k(l)}=\sum_{m=1}^{P} \sum_{n=1}^{R}\left[W_{2, k(l, m, n)}^{2}+W_{4, k(l, m, n)}^{2}\right] .
\end{gathered}
$$

Then the final decision variable is obtained as

$$
Z_{k(l)}^{\mathrm{DC}}=X_{k(l)}-Y_{k(l)}=\sum_{m=1}^{P} \sum_{n=1}^{R} X_{k(l, m, n)}-\sum_{m=1}^{P} \sum_{n=1}^{R} Y_{k(l, m, n)} .
$$

Accordingly, the decision variable $X_{k(l, m, n)}$ of each path obeys a noncentral chi-square PDF with two degrees of freedom, where as $Y_{k(l, m, n)}$ is centrally chi-square distributed with two degrees of freedom. The individual PDFs are given by as follows

$$
\begin{gathered}
f x_{k(l, m, n)}\left(z \mid H_{x}\right)=\frac{1}{2} e^{\left[-\left(z+\lambda_{x}\right) / 2\right]} \cdot I_{0}\left(\sqrt{Z \cdot \lambda_{x}}\right), \\
f Y_{k(l, m, n)}\left(z \mid H_{x}\right)=\frac{1}{2} e^{[-z / 2]}
\end{gathered}
$$

respectively, where $z \geq 0, x=0$ or $1, I_{0}(\cdot)$ is the zero ${ }^{\text {th }}$ order modified Bessel function of the first kind. Let us now express the PDF of the desired user's signal at the output of the acquisition scheme conditioned on the presence of the desired signal in $f x_{k(l, m, n)}\left(z \mid H_{x}\right)$, when communicating over an uncorrelated Rayleigh channel. In this scenario, $E_{c}$ is multiplied by the square of the Rayleigh distributed fading amplitude, $\beta$, which has a chi-square distribution with two degrees of freedom: $f(\beta)=e^{-\beta / \sigma^{2}} / \sigma^{2}$, where $\sigma^{2}$ is the variance of the constituent Gaussian distribution. Then the average pilot signal energy $E c$ per $\mathrm{PN}$ code chip can be expressed as $\overline{E_{c}}=\overline{\beta E_{c}}=\sigma^{2} E_{c}$.

The probability of correct detection for the $l$ th path according to $x=1$ is expressed as

$$
P_{D(l)}^{\mathrm{DC}}=\int_{\theta}^{\infty} f z_{k(l)}^{\mathrm{DC}}\left(z \mid H_{1}\right) d z, \quad \theta \neq 0,
$$

where $\theta=$ threshold value.

$P_{D(l)}^{\mathrm{DC}}=$ the probability of correct detection for the $l$ th path. Finally, the false alarm probability in the context of an $H_{0}$ hypothesis is expressed as

$$
P_{F}^{\mathrm{DC}}=\int_{\theta}^{\infty} f z_{k(l)}^{\mathrm{DC}}\left(z \mid H_{0}\right) d z, \quad \theta \neq 0,
$$

where $P_{F}^{\mathrm{DC}}=$ the false alarm probability.

For comparison, the NC counterpart of the previously described DC scheme is characterized here, where the final decision variable of the $l$ th path is given by

$$
Z_{k(l)}^{\mathrm{DC}}=\sum_{m=1}^{P} \sum_{n=1}^{R}\left\|\frac{1}{\sqrt{2}}\left(\sqrt{\frac{4 E_{c}}{N I_{0} P}} \cdot S_{k(l, m, n)}+I_{k(l, m, n)}\right)\right\|^{2}
$$

where $\|\cdot\|$ represents the Euclidian norm of the complexvalued argument and the factor of $1 / \sqrt{2}$ is employed to normalize the noise variance. The NC decision variable $Z_{K(L)}^{\mathrm{NC}}$ has exactly the same statistical behavior as $X_{k(l)}$ described above. Thus its derivation follows the same procedures that of $f_{X k(l)}(z \mid H x)$ outlined. $S_{k(l, m, n)}$ becomes deterministic while $I_{k(l, m, n)}$ is the complex-valued additive white Gaussian 


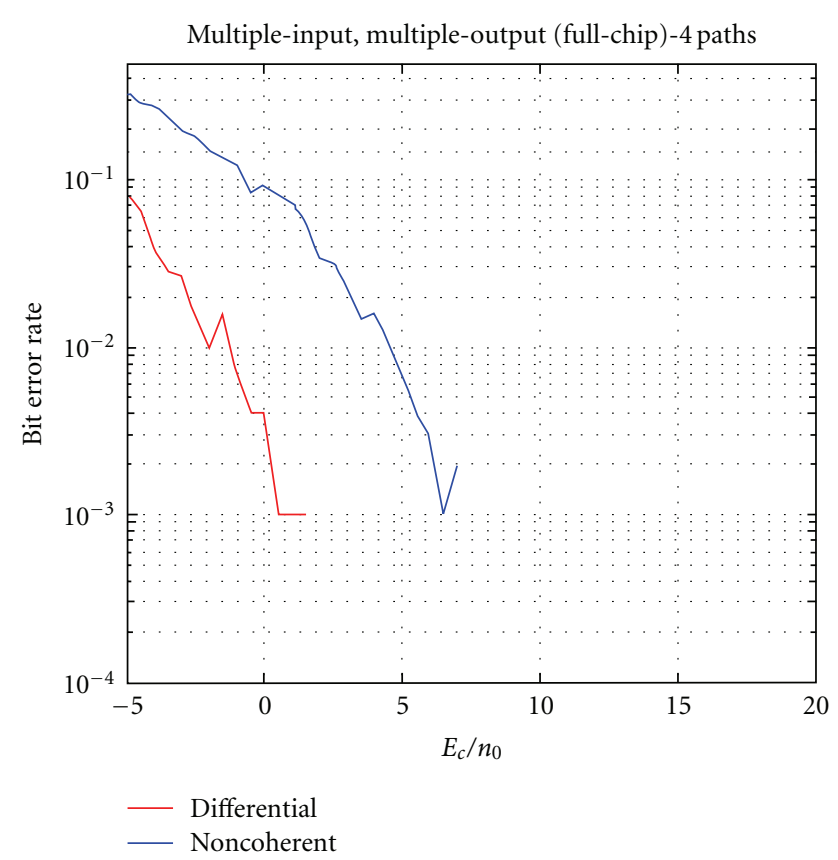

(a) $M * N(1,4)=4$ paths

Multiple-input, multiple-output (full-chip)-16 paths

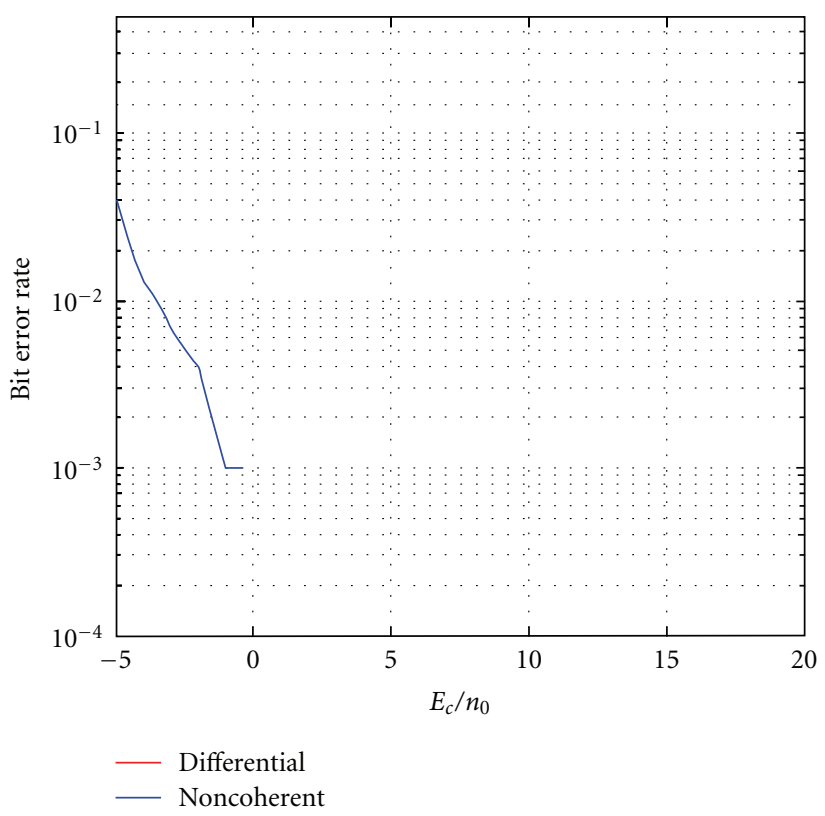

(c) $M * N(4,2)=16$ paths

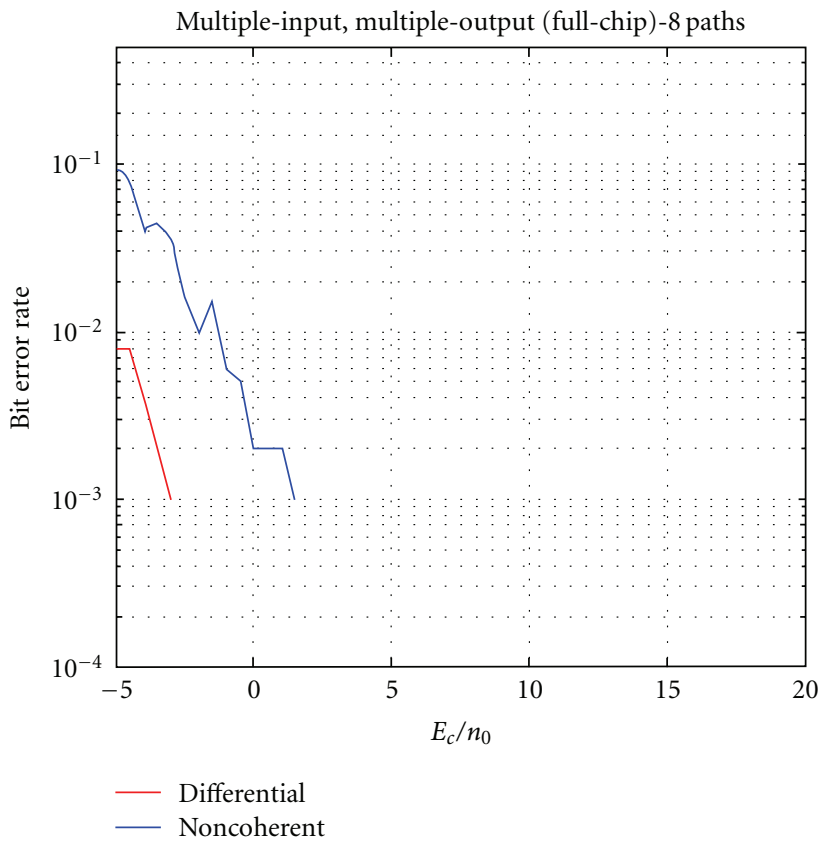

(b) $M * N(2,4)=8$ paths

Multiple-input, multiple-output (full-chip)-32 paths

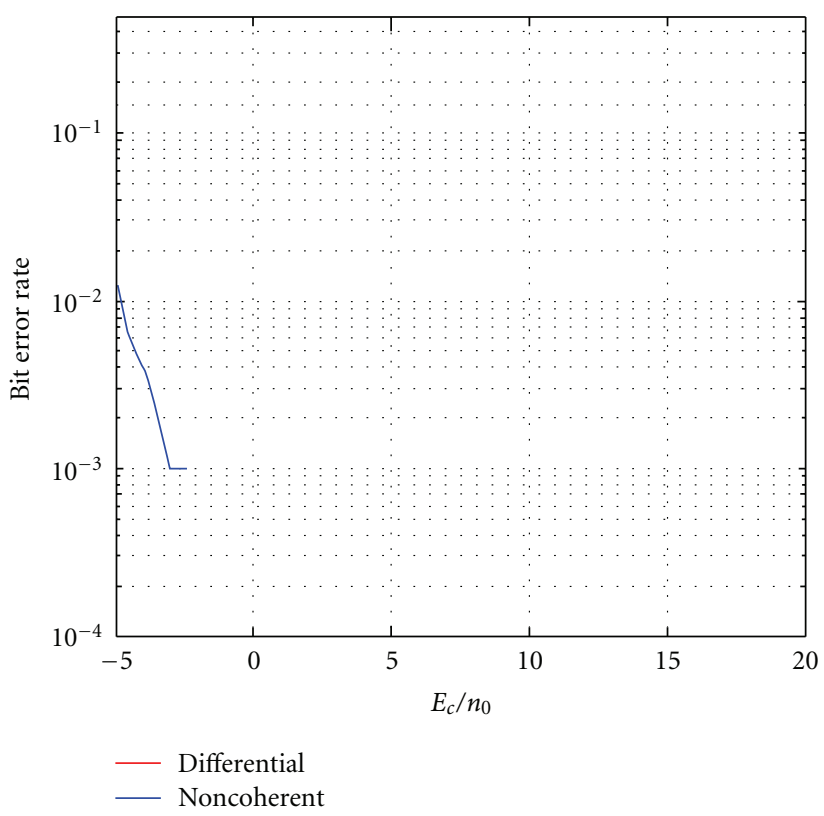

(d) $M * N(8,4)=32$ paths

FIGURE 5: shows the BER characteristics for MIMO full chip using BPSK modulation.

noise having zero means and variances of $\sigma^{2}=2$ for both their real and imaginary parts.

The probability of correct detection corresponding to $x=1$ for the $l$ th path is obtained as

$$
P \frac{\mathrm{NC}}{D(l)}=e^{-\theta / \mu_{1}} \cdot \sum_{k=0}^{P \cdot R-1} \frac{\left(\theta / \mu_{0}\right)^{k}}{k^{\prime}}
$$

where $k=$ False locking penalty factor, $\theta=$ threshold value
In explicit MAT analysis, formulas are provided as singleantenna-aided serial search-based code acquisition system. There is no distinction between a single-antenna-aided scheme and a multiple-antenna-assisted one in terms of analyzing their MAT performance, except for deriving their correct detection and the false alarm probability based upon using transmit/receive antennas. It is assumed that in each chip duration $T_{c}, \alpha$ number of timing hypotheses are tested, which are spaced by $T_{c} / \alpha$. Hence, the total uncertainty 

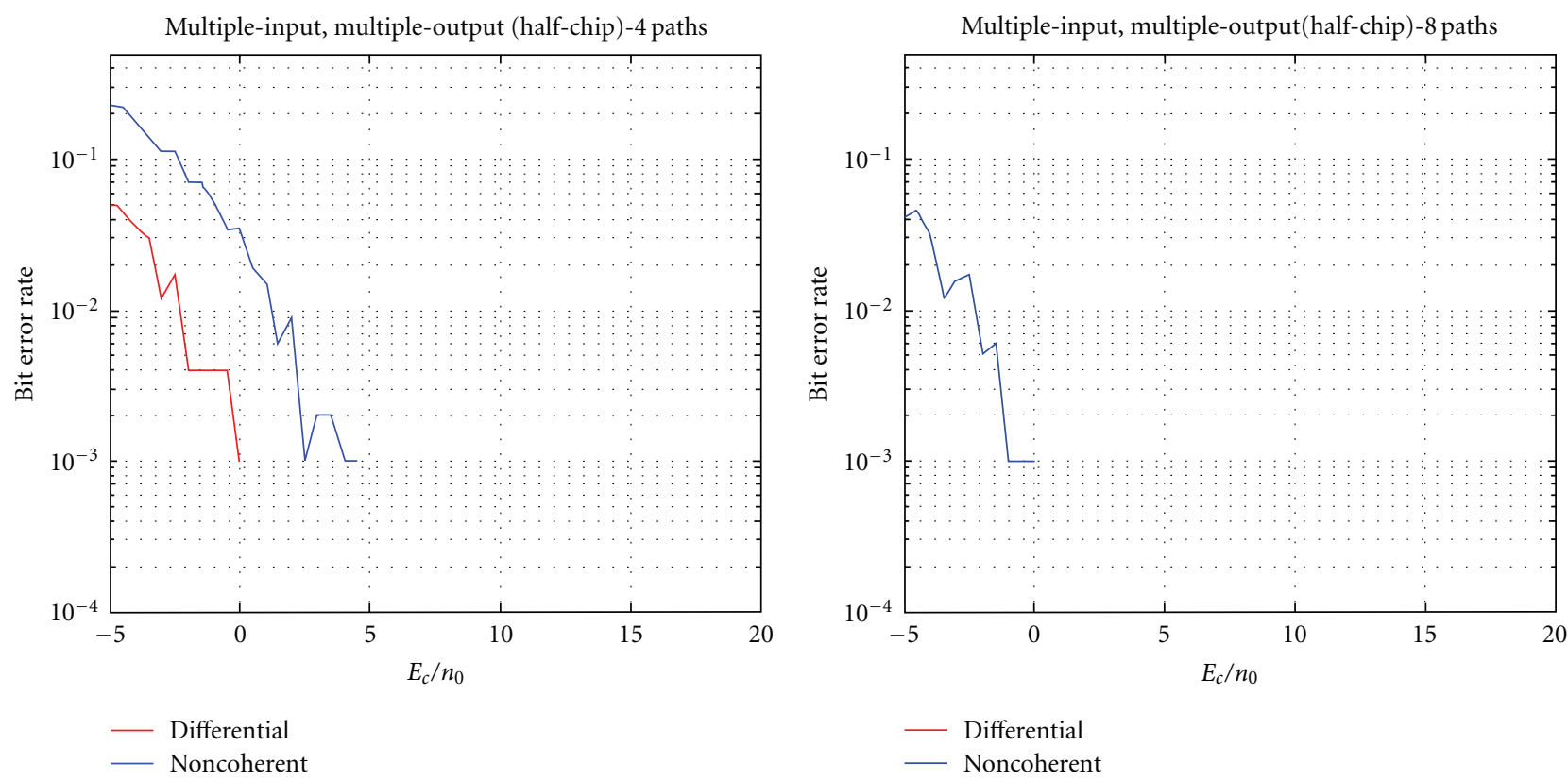

(a) $M * N(1,4)=4$ paths

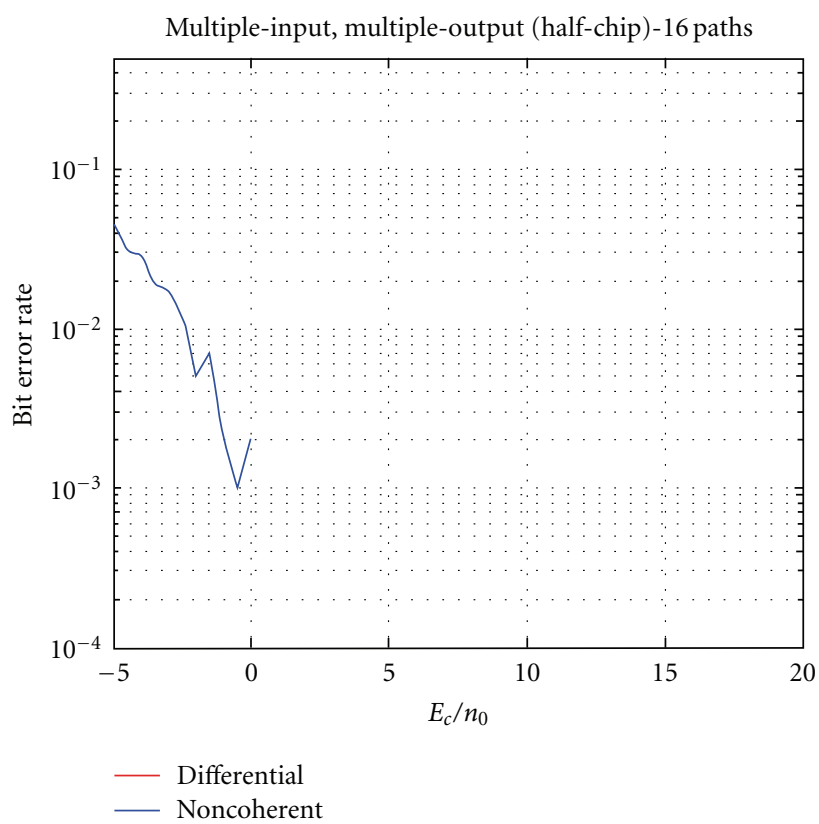

(c) $M * N(4,2)=16$ paths



(d) $M * N(8,4)=32$ path

FIGURE 6: BER characteristics of MIMO receiver simulation half chip using BPSK modulation.

region is increased by a factor of $\alpha$ [8], while the analysis of the MAT performance of NC receiver $Z_{\text {tot }}$ decision variable is generated by the NC module and is compared with threshold value $\theta_{1}$. The search method involves two serial searches, namely search mode and validation mode and hence called as Double Dwell Serial Search method. When the desired user tentative code phase is obtained in the search mode of double dual serial search, the verification mode is activated which may use DC/NC modules in order to conform that the correct code phase is same as in search mode. DC scheme is excluded in the search mode since it requires further processing carried out with DC module and thus the complexity is minimized, by not employing DC scheme to verification mode. Let $Z_{1}$ and $Z_{2}$ be the two decision variables of search and verification modes respectively and $\theta_{1}$ and $\theta_{2}$ be acquisition threshold of search and verification mode respectively. $Z_{1}$ is compared to $\theta_{1}$, and if it exceeds the threshold, $Z_{2}$ generated by either $\mathrm{DC}$ or $\mathrm{NC}$ module is compared to $\theta_{2}$. If successful code acquisition is declared, then the code tracking loop is enabled. Otherwise, the 
Multiple-input, multiple-output (full-chip)-4 paths

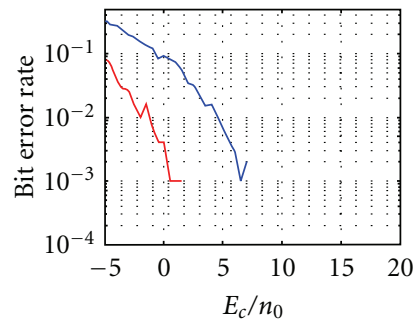

(a)

Multiple-input, multiple-output (half-chip)-4 paths

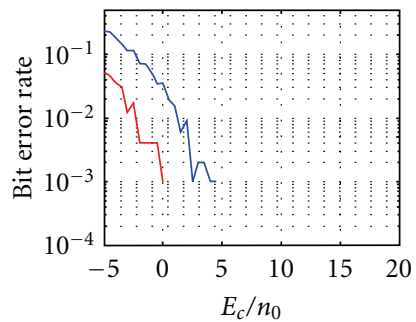

- Differential

- Noncoherent

(e)
Multiple-input, multiple-output (full-chip)-8 paths

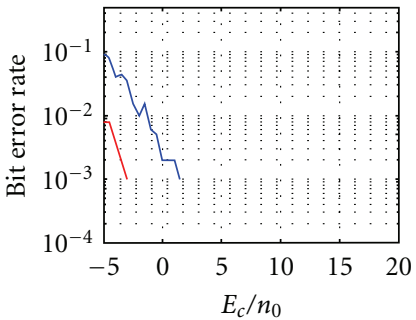

(b)

Multiple-input, multiple-output (half-chip)-8 paths

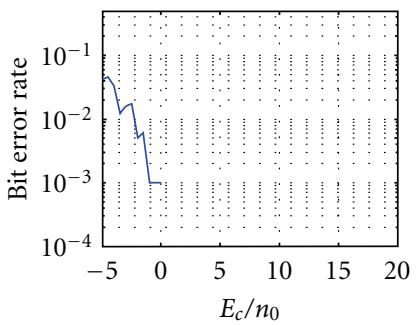

- Differential

(f)
Multiple-input, multiple-output (full-chip)-32 paths

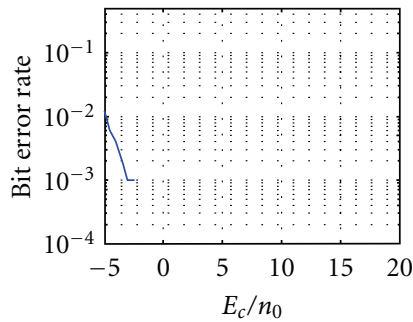

(c)

Multiple-input, multiple-output (half-chip)-16 paths

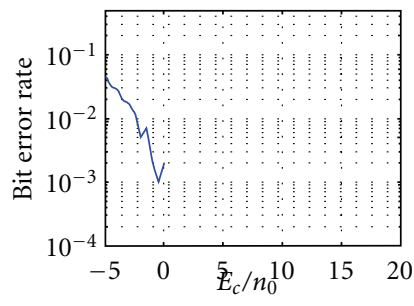

- Differential

(g)



(d)

Multiple-input, multiple-output (half-chip)-32 paths

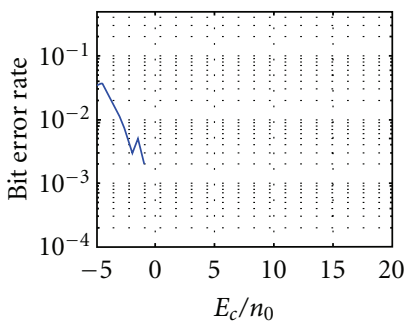

- Differential

(h)

FIgURE 7: Comparison between MIMO full chip (Figures 7(a)-7(d)) and half chip configuration (Figures 7(e)-7(h)).

acquisition system reverts back to the search stage, until the correct code and its phase are found.

\section{Simulation Results and Discussions}

The simulation was carried out at RF signal processing lab. Initially single-input single-output (SISO) system was considered employing DC and NC schemes using BPSK modulation scheme with full chip rate, and half chip rate, respectively. It is evident that DC code acquisition system performs better in terms of BER characteristics when compared to NC systems. This is as shown in Figures 4(a) and $4(\mathrm{~b})$.

The Figures 5(a), 5(b), 5(c), and 5(d) shows the MIMO receiver simulation for $4,8,16$, and 32 path employing DC and NC code acquisition for full chip rate using BPSK modulation method. In Figures 5(c) and 5(d), it is seen that BER is nil and also is DC is a better option than NC. Further it is also evident that BER decreases as transmission path increases hence improving the capacity.

Similarly Figures 6(a), 6(b), 6(c), and 6(d) depict the MIMO receiver simulation for $4,8,16$, and 32 path employing DC and NC code acquisition for half chip rate using BPSK modulation method. In the Figures 6(a) and 6(d), BER is reduced much comparing to full chip. This shows that DC is better suited when compared to NC code acquisition method. Also it is observed that in half chip performance characteristics the BER decreases with increase in the number of transmission path.

For the benefit of visualization and the comparison effects, the four sets of figures are shown again in Figure 7, Which brings out the difference between MIMO full chip (Figures $7(\mathrm{a})-7(\mathrm{~d})$ ) and half chip configuration (Figures $7(\mathrm{e})-7(\mathrm{~h}))$.

Figure 8 shows the variation of the mean acquisition time(MAT) with respect to the $\operatorname{SINR}\left(E_{c} / n_{0}\right)$ ratio. From the figures, it is clear that the MAT decreases with increase of SINR ratio. This is one of the favorable results of this system. The mean acquisition time of DC is less than NC code acquisition. From the result, it is observed that as SINR ratio is increased, the mean acquisition time is reduced considerably for both DC and NC code acquisition methods. Also For 32 paths, the MAT of DC code acquisition is very less or zero. The MAT for DC code acquisition is decreasing as the number of transmission path increases. Hence DC is again a better option than NC.

\section{Conclusion}

This paper is a successful implementation of full chip and half chip rate with DC and NC schemes. It is evident that MIMO technique is employed with spread spectrum to increase capacity and improves performance in the MIMO scheme. This paper highlights the effects of two performance metrics for MIMO-DSCDMA over uncorrelated Raleigh 


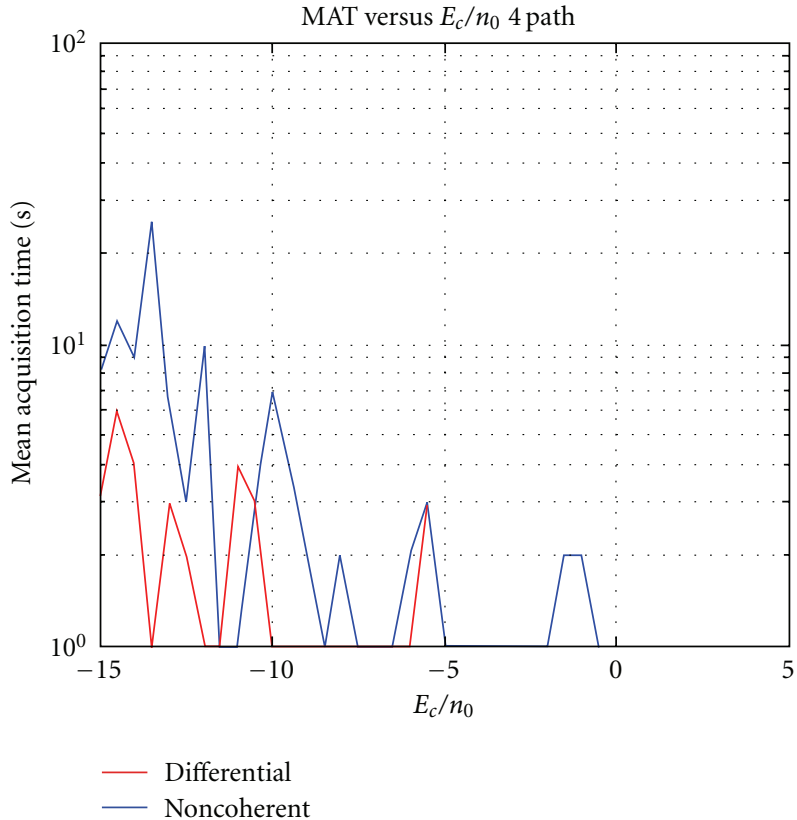

(a) $M * N(1,4)=4$ path

MAT versus $E_{c} / n_{0} 32$ path

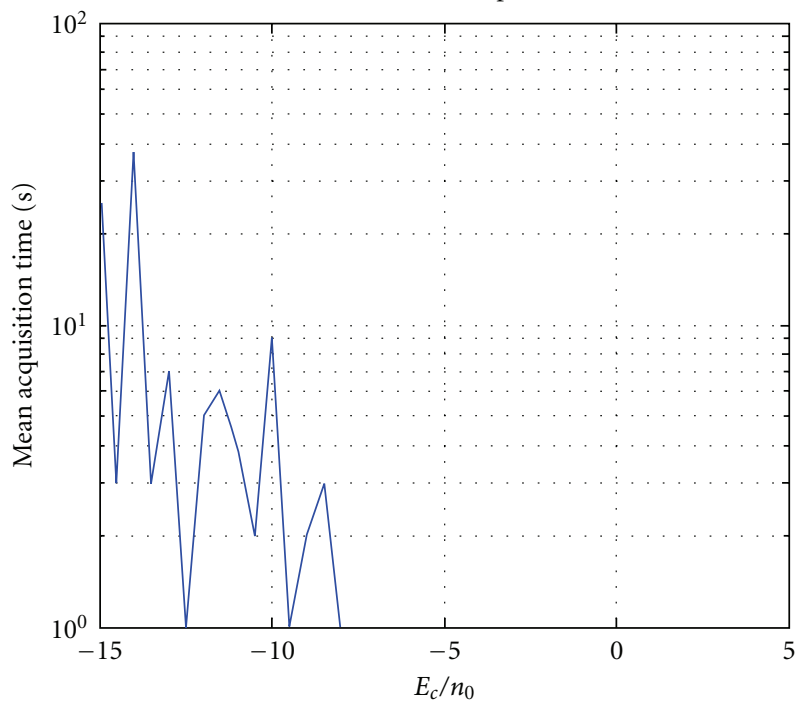

- Differential

- Noncoherent

(c) $M * N(4,4)=16$ path

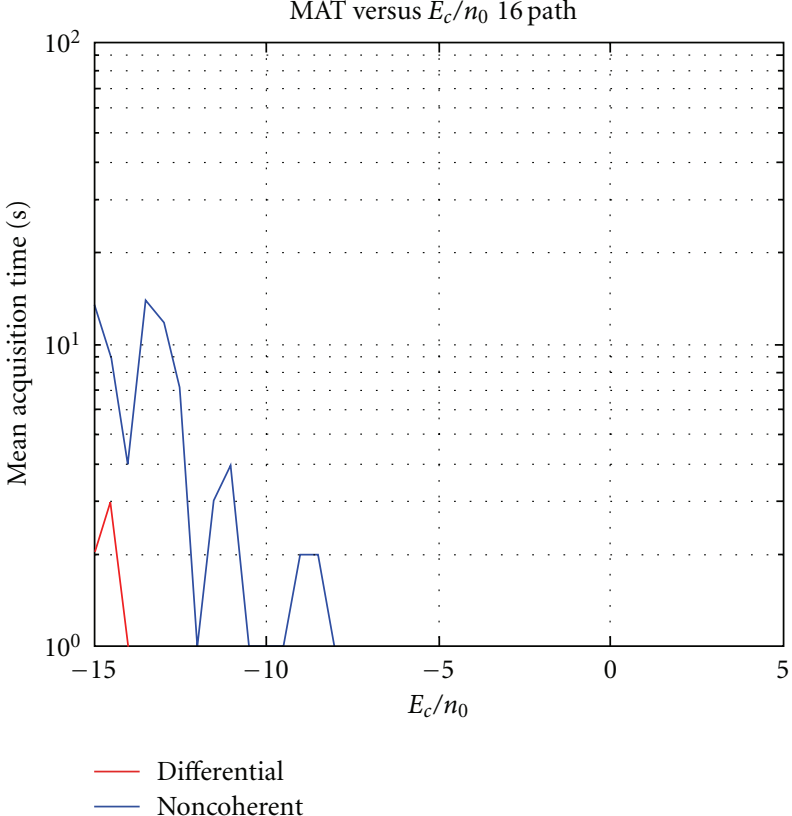

(b) $M * N(2,4)=8$ path

MAT versus $E_{c} / n_{0} 8$ path



(d) $M * N(8,4)=32$ path

FIGURE 8: Mean acquisition time versus SINR characteristics-MIMO.

wireless channel, namely, BER characteristics and MAT performance characteristics using Matlab toolbox. The simulation results are compared for four MIMO systems for both DC and NC code acquisition schemes. The observations from the simulation results are as follows:

(i) As the number of paths increases, the BER is decreased for both DC code acquisition and NC code acquisition for both the half and full chip rate schemes. (ii) DC performance is better than NC in both half chip and full chip for both SISO and MIMO.

(iii) Half chip method is best suited in terms of BER when compared to full chip for both DC and NC schemes.

(iv) As the number of paths increases the BER is decreased for both half chip and full chip.

(v) The mean acquisition time of DC is less than NC code acquisition. 
(vi) As SINR ratio is increased, the mean acquisition time is reduced considerably for both DC and NC code acquisition methods.

(vii) For 32 paths, the MAT of DC code acquisition is very less or zero stating that the mean acquisition time is reduced as the number of path increases.

\section{Acknowledgments}

The authors express their sincere thanks to The management, The Director (Academics), The Principal, The Head of the Department ECE, Sri Ramakrishna Engineering College, coimbatore-22, TN, India for providing the resources to carry out this research with constant encouragement. The authors also express their sincere thanks to the doctoral committee members Dr. R. Rangarajan, The Dean Dr. Mahalingam, college of Engineering and technology, Dr. Shankaranarayanan, The Dean EASA College of Engineering for their constant support and technical guidance.

\section{References}

[1] M. R. Bhatnagar, R. Vishwanath, and V. Bhatnagar, "Performance analysis of space-time block codes in flat fading MIMO channels with offsets," EURASIP Journal on Wireless Communications and Networking, vol. 2007, Article ID 30548, 7 pages, 2007.

[2] C. D'Amours and A. O. Dahmane, "Spreading code assignment strategies for MIMO-CDMA systems operating in frequencyselective channels," EURASIP Journal on Wireless Communications and Networking, vol. 2009, Article ID 839424, 13 pages, 2009.

[3] K. Simon, J. K. Omura, R. A. Scholtz, and B. K. Levitt, Spread Spectrum Communications Handbook, chapter 1, Tata McGrawHill Publications, New Delhi, India, 2001.

[4] W. Suwansantisuk and M. Z. Win, "Multipath aided rapid acquisition: optimal search strategies," IEEE Transactions on Information Theory, vol. 53, no. 1, pp. 174-193, 2007.

[5] L. L. Yang and L. Hanzo, "Serial acquisition of DS-CDMA signals in multipath fading mobile channels," IEEE Transactions on Vehicular Technology, vol. 50, no. 2, pp. 617-628, 2001.

[6] P. Greisen, S. Haene, and A. Burg, "Simulation and emulation of MIMO wireless baseband transceivers," EURASIP Journal on Wireless Communications and Networking, vol. 2010, Article ID 196796, 12 pages, 2010.

[7] W. Suwansantisuk, M. Z. Win, and L. A. Shepp, "On the performance of wide-bandwidth signal acquisition in dense multipath channels," IEEE Transactions on Vehicular Technology, vol. 54, no. 5, pp. 1584-1594, 2005.

[8] N. Sathish kumar and K. R. Shankar Kumar, "A novel approach of NC and DC code acquisition in MIMO assited DS-CDMA wireless channel," Journal of Computing, vol. 2, no. 11, pp. 2151-9617, 2010. 

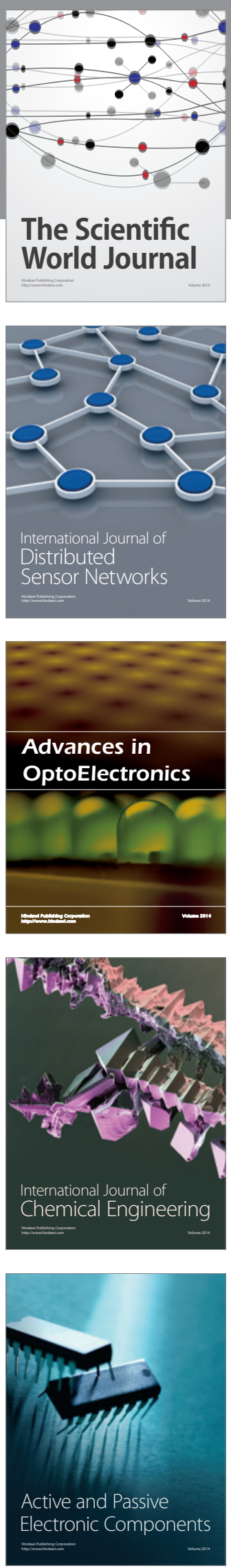
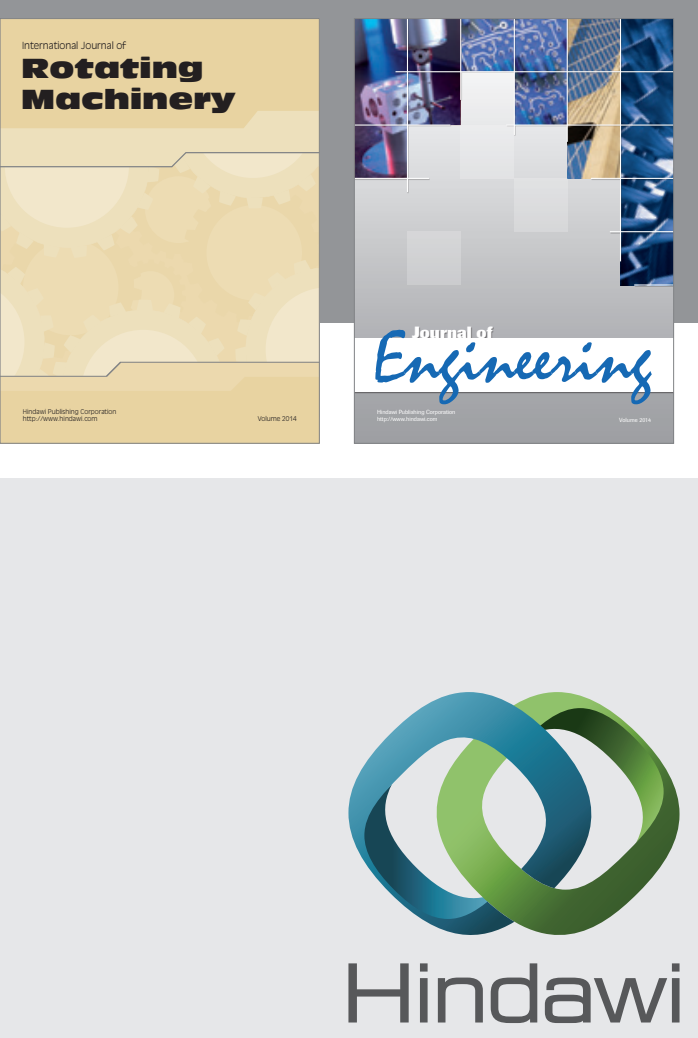

Submit your manuscripts at

http://www.hindawi.com
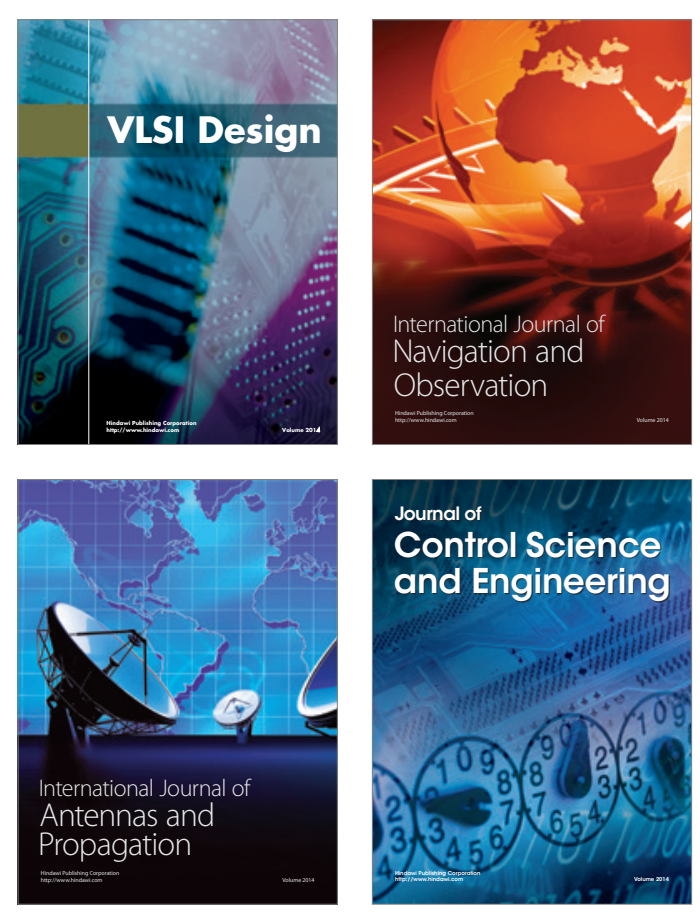
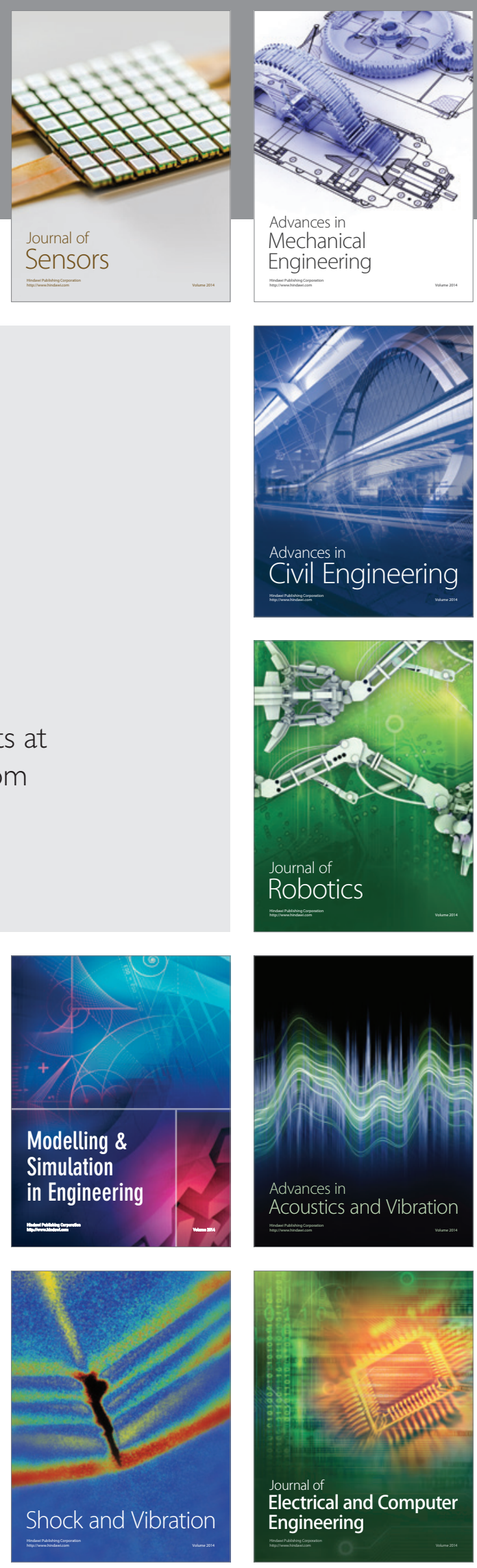